Research Article

\title{
Proactive VNF Scaling with Heterogeneous Cloud Resources: Fusing Long Short-Term Memory Prediction and Cooperative Allocation
}

\author{
$\operatorname{Ran} \mathbf{X u}(\mathbb{D}$ \\ State Key Laboratory of Networking and Switching Technology, Beijing University of Posts and Telecommunications, \\ Beijing, China \\ Correspondence should be addressed to Ran Xu; ranxu@bupt.edu.cn
}

Received 26 June 2019; Revised 25 August 2019; Accepted 18 November 2019; Published 28 January 2020

Academic Editor: Alessandro Lo Schiavo

Copyright (c) 2020 Ran Xu. This is an open access article distributed under the Creative Commons Attribution License, which permits unrestricted use, distribution, and reproduction in any medium, provided the original work is properly cited.

Network function virtualization (NFV) is designed to implement network functions by software that replaces proprietary hardware devices in traditional networks. In response to the growing demand of resource-intensive services, for NFV cloud service providers, software-oriented network functions face a number of challenges, such as dynamic deployment of virtual network functions and efficient allocation of multiple resources. This study aims at the dynamic allocation and adjustment of network multiresources and multitype flows for NFV. First, to seek a proactive approach to provision new instances for overloaded VNFs ahead of time, a model called long short-term memory recurrent neural network (LSTM RNN) is proposed to estimate flows in this paper. Then, based on the estimated flow, a cooperative and complementary resource allocation algorithm is designed to reduce resource fragmentation and improve the utilization. The final results demonstrate the advantage of the LSTM model on predicting the network function flow requirements, and our algorithm achieves good results and performance improvement in dynamically expanding network functions and improving resource utilization.

\section{Introduction}

Network function virtualization (NFV) plays an important role in future network innovation, which breaks the ossified and bloat of the network [1]. The purpose of NFV is to replace the network functions (NFs) such as firewalls and load balancers with software on existing commercial devices through virtualization technology, thus replacing the expensive and dedicated hardware (middlebox) in traditional networks [2].

In recent years, with the emergence of smart devices and applications, communication networks have gradually merged into large-scale cloud architecture networks. More and more applications have transferred the necessary computing load to the cloud platform, which has greatly reduced the pressure on individual users $[3,4]$. Recently, NEC/Netcracker has unveiled the NFV cloud marketplace by introducing a Network-as-a-Service solution [5]. While cloud-based applications can alleviate user hardware or software requirements and workloads, outsourcing network functions packet processing to the cloud leave the complex scheduling and provisioning services to the NFV providers $[6,7]$. Hence, in the context of NFV, achieving high network resource utilization and meet Service Level Objective (SLO) for cloud service providers is urgent and means high profit returns $[8,9]$.

Usually, in NFV service chains [10], NFs have various resources requirement that includes traffic-intensive, computationally intensive, and memory-intensive. For example, intrusion detection systems and secure encryption systems require sufficient CPU resources, while software routers rely more on memory resources, but network input and output devices rely more on bandwidth resources [11, 12]. In addition to simple constraints, combinatorial constraints are also possible, and there are various job flows in cloud services, for example, long-term job flows and short-term job lows coexist, or requiring two tasks to be placed on two distinct machines, and so on $[12,13]$. Different NFs are now 
consolidated on the same physical server [14]. However, in the cloud center, the cluster machines that serve network flows are heterogeneous, and one of the configuration examples is shown in Table 1 [6]. Such heterogeneity would make accurate visibility into future resource demands difficult, and it will reduce the effectiveness of traditional slotbased scheduling $[6,7]$.

In order to obtain better quality assurance and costeffectiveness of traffic services, there have been some research results. For example, in the research results of [15], the authors combined online learning and optimization, designed a proactive forecast of upcoming traffic demand, and actively adjusted VNF (virtual network functions) deployment. Also in the research results of [7], the authors actively predict the service chain demand of flow through online learning, adjust the instances of deployment of NFV cloud service providers according to the prediction, and solve the problem of new instance allocation and service chain routing. In addition to the research results of [16], the researchers provided an online algorithm for dynamic VNF deployment and placement with minimal design cost for cloud data centers of NFV service providers, and another scheme [17] scales VNF resources vertically on the fly, which is not encouraged in most cases as it requires rebooting the system [7], and so on.

However, previous studies ignored the diversity of network function resource requirements and the heterogeneity of actual server resource configuration, which can easily result in resource fragmentation and hence low resource utilization [6,12]. Deep learning is a branch of machine learning that attempts to learn high-level features directly from the original data [18]. Inspired by the successful use of deep learning on traffic flow [19], in this paper, we use long short-term memory recurrent neural network (LSTM RNN) to predict the customer service chain requests and the size of the flow in the NFV network. With prediction results, we derive the traffic classification and processing capacity requirement. Then, we deploy virtual network functions on different virtual machines and collaborative allocation of multiple resources according to the demand of different types of resources.

We summarize the contributions of this work in the following:

(1) We investigate the VNF provisioning and resource allocation problem by LSTM RNN model to predict the type and amount of resource requirements, and LSTM RNN adapts well to the requirement of timevarying demands; hence, we can dynamically allocate the corrected amount of resource on the heterogeneous cluster machines for NFV cloud providers.

(2) We offer a collaborative resource allocation approach on different resource types, which reduces the resource fragmentation and increases the resource utilization.

(3) We evaluate our model on a real data set, and the correctness and efficiency of our method and analysis are validated by simulation studies.
TABle 1: Normalized CPU and memory configuration in Google Machine Cluster.

\begin{tabular}{lcc}
\hline Machine number & CPU & Memory \\
\hline 6732 & 0.50 & 0.50 \\
3863 & 0.50 & 0.25 \\
1001 & 0.50 & 0.75 \\
795 & 1.00 & 1.00 \\
126 & 0.25 & 0.25 \\
52 & 0.50 & 0.12 \\
5 & 0.50 & 0.03 \\
5 & 0.50 & 0.97 \\
3 & 1.00 & 0.50 \\
1 & 0.50 & 0.06 \\
\hline
\end{tabular}

\section{Problem Description and Model}

2.1. Problem Description. The cluster machines are heterogeneous, and that different NFs have different sensitivities to resource requirements; the goal is to improve the utilization of multiresources. Therefore, we can describe the problem as follows: given a certain amount of resources (for example, $\mathrm{CPU}$, memory, etc.) and resource requirements of each NFV service chain job, how to configure network functions and allocate resources to jobs to achieve high utilization is under the consideration of heterogeneous resource configuration.

For each request flow, it is necessary to ensure the reasonable allocation of resources. Traditionally, simply balancing the load of traffic results in inefficiencies and limits the processing power of the entire NFV cluster. Intuitively, the average load of all resources can be used as a solution. However, the problems that will exist include the following: it does not combine the actual configuration of the servers and it will take resource fragmentation; besides, much information about request network flow is not to be learned and considered (required type, traffic volume, and so on) $[20,21]$.

For example, as shown in Figure 1, two VMs (virtual machines) in the server have three different resources that include CPUs, memory, and bandwidth, and the angle brackets shown in the figure represent the service request vector, where the values correspond to the three required resources, respectively. Because of the flexibility of NFV, different network functions can be deployed on the same server. Considering this situation, the network function required for service 1 is deployed in VM1, the network function required for service 2 is deployed in VM2, and both of the VMs have the network function for flow 3. As shown in the figure, they have an average load of 0.25 and 0.25 , respectively, which is the same. Therefore, when allocating the resources required by service 3 from the perspective of average allocating, both VM1 and VM2 have the same selected condition. However, the VM1 is more nervous because its CPU resources are insufficient. Hence, it is impossible to allocate resources for the next request, and VM1 forms an unusable resource fragment. Furthermore, from a deployment point of view, if we can predict the required functions and resource requirements of service 3 , deploy the network functions which is required by service 3 


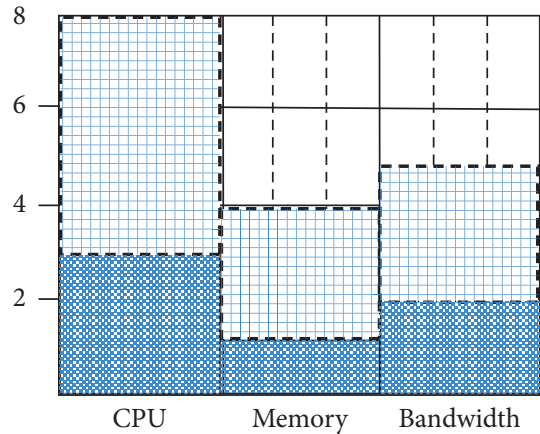

Demand $<1,3,2>$

Job 1

Demand $<3,1,2>$

Job 2

Demand $<5,3,3>$

Job 3

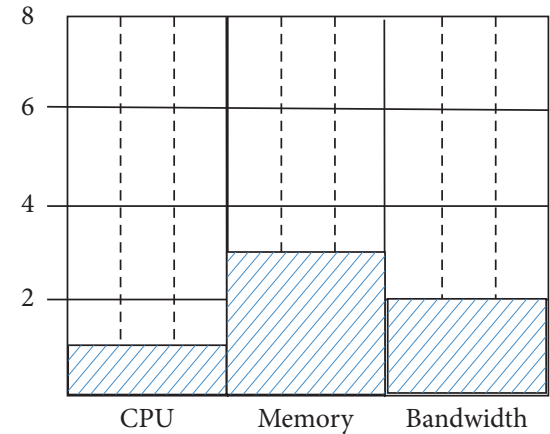

Demand $<1,3,2>$

Job 1

Demand $<3,1,2>$

Job 2

Demand $<5,3,3>$

Job 3

(a)

(b)

FIGURE 1: Examples of complementary types of different resources. (a) Server 1. (b) Server 2.

on VM2; together with the resource allocation complementary considerations, the resource utilization of VM2 will be improved, while the VM1 can be reserved for a higher rate of available resources.

Furthermore, because of the heterogeneity of server resources and diverse flow requirements, the complexity of resource assignment is increased. As shown in Figure 2, the same value is used to represent the different resource capacities of the server, and $t$ is used to represent the unit time period. The coordinated allocation of various resources is considered in Figure 2, and the resources are reserved for subsequent requests in a reasonable manner without considering the characteristics of the flows. However, considering the life cycle of each service flow, if there are service requests with more than 3 memory unit resources in the next eight time periods, such as request $\langle 3,4,2\rangle$, it will be unable to service request and refuse. Actually, after a period of time, the sum of the remaining resources of the server is sufficient to satisfy the user's request. So when we allocate resources, by combining the consideration of the traffic flow characteristics, although one unit of CPU and bandwidth resources is wasted in server 1 , the service provider can receive more services in the next 7 time periods. That is to get more revenue while meeting customer service level goals.

In brief, cloud service resources are expensive and critical to the business. Different network function instances are used as intermediate processing nodes, and all applications need to meet specific requirements and constraints. How can we design solutions that dynamically scale VNF instances and coordinate them onto servers, to adequately serve fluctuating input traffic and to achieve long-term goals while meeting short-term resource needs over the long run of the system? The problem is the high variant of a multidimensional bin packing problem, which is NP-hard [22].
2.2. Network and Flow Model. In the problem model studied, we use $S$ to represent the network traffic flow set. $|S|$ is used to denote the number of service set used and $[1,|S|]$ represents the set of integers from 1 to $|S|$, and similar representations are analogized in this way. Thus, the service chain can be described as $s_{i}$, which corresponds network service $i$. $F$ represents the virtual network function set and $f_{i j}=1$ represents the virtual network function $i$ corresponding to the network service $j$. In addition, for the server set $L$, $l \in[1,|L|]$ denotes the server node $l$. The corresponding capacity of various resource sets $R$ are represented by $C_{l}=\left\{c_{l}^{1}, c_{l}^{2}, \ldots, c_{l}^{r}\right\}$. Because different server nodes can be equipped with different virtual network functions, we define the node function set $V_{l}=\left\{v_{u}, v_{w}, \ldots, v_{v}\right\}$ equipped with the server as a subset of the virtual network function set $F=\left\{f_{1}, f_{2}, \ldots, f_{f}\right\}$. For example, the network function that can be processed with the server node $l$ is denoted as $\left\{f_{1}, f_{5}, f_{8}\right\}$.

2.3. Problem Model. In the multiresource problem of NFV cloud service, for each network service request $s_{i}$, we define $f_{l i}=1$ as the required network functions to be processed by the server $l$ and denote the number of required resources of $r$ as $d_{i j r}$. Therefore, the objective function of resource utilization rate $u_{l}$ of server $l$ can be expressed as follows:

$$
u_{l}=\sum_{i=1}^{|S|} \sum_{r=1}^{|R|} \frac{f_{l i} d_{i j r}}{c_{r}} .
$$

Hence, in $T=\{1,2, \ldots, T\}$ time periods, the total demand of service chain is

$$
U=\sum_{t=1}^{|T|} \sum_{l=1}^{|L|} u_{l}(t) .
$$




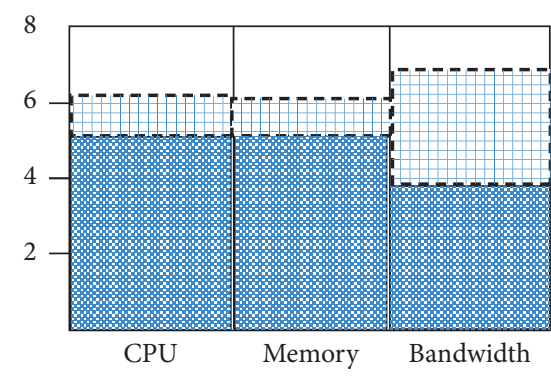

Demand $<1,1,3>$

Job 1

Life time $8 \mathrm{t}$

Demand $<5,5,4>$

Job 2 \%r䅦

Life time $8 \mathrm{t}$

Demand $<2,3,3>$

Job 3

Life time $1 \mathrm{t}$

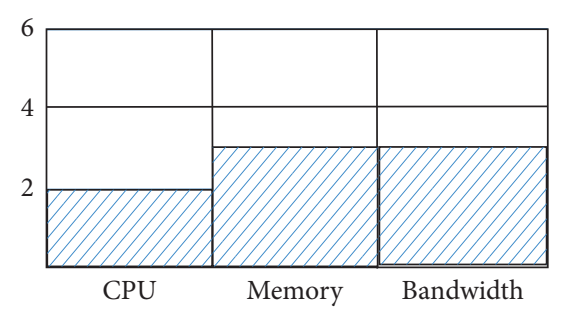

Demand $<1,1,3>$

Job 1

Life time $8 \mathrm{t}$

Demand $<5,5,4>$

Job 2 \%

Life time $8 \mathrm{t}$

Demand $<2,3,3>$

Job 3

Life time $1 \mathrm{t}$

Figure 2: Examples of complementary types of different flow types. (a) Server 1. (b) Server 2.

When processing the function requests, in order to ensure that the scheduled resources of the network service meet the existing network conditions, the restricted conditions are expressed by equations (3) to (5). In the constraint condition (3), it is necessary to ensure that the capacity of various resources allocated to the service set should not exceed the total available resources. We define the binary variable $x_{i j k}=1$ as the function $j$ corresponding to the service chain $i$ processed by the virtual machine $k$. Condition (4) has to ensure that the required network function in the service flow $s_{i}$ is processed by at least one node. Equation (5) introducing variable $y_{u v}^{i}=1$ which represents directed edge $(u, v)$ belongs to the assigned source path, and it is used to ensure that the function node selected by the service chain is loop-free, where $z_{u v}^{i}$ is defined as the order number on the path. $N_{p}^{s}$ is the subset of the selected path node set $N_{p}$ and $h$ is an integer greater than or equal to 2 less than $\left|N_{p}\right|$ :

$$
\begin{aligned}
& \text { s.t. } \sum_{i \in[1,|S|]} d_{i j r}, \quad f_{l i} \leq c_{r}, f_{j} \in V_{l}, \forall r \in R, \forall l \in L . \\
& \sum_{k \in L} x_{i j k} \geq 1, \quad \forall i \in[1,|S|], j \in\left[1,|F|_{i}\right] . \\
& \sum_{u v \in E} z_{u v}^{i} y_{u v}^{i}-\sum_{v w \in E} z_{v w}^{i} y_{v w}^{i}=0, \quad \forall i \in[1,|S|], \\
& \forall u, v, w \in N_{p}^{s}\left|N_{p}^{s}\right|=h, \quad 2 \leq h \leq\left|N_{p}\right| .
\end{aligned}
$$

\section{Method Design}

3.1. Long Short-Term Memory (LSTM). Neural networkbased prediction is able to nonlinearly model and approximate any continuous function to any desired accuracy theoretically [23]. With the development of artificial intelligence, deep learning approaches have been gradually shifted to the computational intelligence forecast approaches. Among them, RNN enables to capture the features of time series and maps the input of sequential data and the output, which is naturally suitable as the method to capture the temporal evolution of flow. Then, long short-term memory (LSTM) was developed to solve the problem of gradient vanishing along with the RNN sequence [24]. The basic unit of LSTM is memory block instead of traditional neuron node. Each memory block contains a set of recurrently connected subnets and three multiplicative units: the input, the output, and forget gates, which provide continuous analogues of write, read, and reset operations on the cells. Against recurrent cell, the LSTM cell adds the forget gate, and the forget gates allow LSTM cells to remove or add information over long periods of time.

The evolution of flow can be considered as a temporal process. The incoming request traffic flow at the the time interval is denoted by $a_{i}(t)$. At time $T$, the task is to predict traffic flow $a_{i}(T+1)$ based on the historical sequence $A=\left[a_{i}(t) \mid t=1,2, \ldots, T\right]$. Flow can be classified into long term and short term according to the definition. Suppose that the input historical traffic flow sequence is denoted as $X=\left(x_{1}, x_{2}, \ldots, x_{T}\right)$, the following equations should be iterated to compute the hidden vector sequence $h=\left(h_{1}, h_{2}, \ldots, h_{T}\right)$ and the output predicted flow sequence $Y=\left(y_{1}, y_{2}, \ldots, y_{T}\right)$ by the LSTM network:

$$
\begin{aligned}
& h_{t}=H\left(W_{x h} x_{t}+W_{h h} h_{t-1}+b_{h}\right), \\
& y_{t}=W_{x h} h_{t}+b_{y},
\end{aligned}
$$

where $H$ is the hidden layer function, $W$ denotes the input hidden weight matrix, and $b$ denotes the bias vector sigmoid function, and $H$ is implemented by the following composite function: 


$$
\begin{aligned}
& \text { The input: } p_{t}=\tanh \left(W_{c}\left[h_{t-1}, x_{t}\right]+b_{c}\right) \\
& \text { The input gate: } i_{t}=\operatorname{sigmoid}\left(W_{i}\left[h_{t-1}, x_{t}\right]+b_{i}\right) \\
& \text { The forget gate: } f_{t}=\operatorname{sigmoid}\left(W_{f}\left[h_{t-1}, x_{t}\right]+b_{f}\right) \\
& \text { The output gate: } o_{t}=\operatorname{sigmoid}\left(W_{o}\left[h_{t-1}, x_{t}\right]+b_{o}\right) \\
& \text { The state: } c_{t}=f_{t} \cdot c_{t-1}+i_{t} \cdot p_{t} \\
& \text { The output: } h_{t}=o_{t} \cdot \tanh c_{t}
\end{aligned}
$$

The mean absolute error (MAE) is used as the loss function, which is given by the following equation:

$$
\text { MAE }=\frac{1}{n} \sum_{i=1}^{n}\left(y_{i}-\widehat{y}_{i}\right),
$$

where $\hat{y}_{i}$ represents the predicted value and $y_{i}$ denotes the actual value. The prediction evaluation metric uses the common RMSE (root mean square error):

$$
\text { RMSE }=\sqrt{\frac{1}{n} \sum_{i=1}^{n}\left(y_{i}-\hat{y}_{i}\right)^{2} .}
$$

By training the model of learning, we predict the type and size $a_{i}(t)$ of the flows that arrive in the next time slot $t+1$ at each specified time point $t$. Because the source and destination of each flow are fixed, we can assume that the required functions are predicted by the source and destination pairs. After that, the predicted result is used to deploy the newly launched VNF according to the current server resource configuration and usage. The allocation algorithm is used to solve the multidimensional bin packing problem and the request instances and remaining resources that change over time. In our deployment design, a heuristic approach is taken to minimize the resource ratios of the remaining nodes that satisfy the condition, while allowing other nodes to have more available space capacity for allocating other new instances. It is similar to relax the balance criteria increasing the utilization levels in [25], and the difference is that the aim of the candidate is minimizing resource fragmentation. The selected reference standard for deployment can be expressed as

$$
P_{l}=\sum_{r=1}^{|R|} \frac{\mathrm{Re}_{l}^{r}}{M_{r}}
$$

where $\operatorname{Re}_{l}^{r}$ is the remaining resources of class $r$ on server $l$ and $M_{R}$ is the maximum value of the remaining resources on the server. The following is a simple example. It is assumed that the remaining resources of server $\mathrm{A}$ are configured as $\langle 10,6,2\rangle$, the remaining resources of server $B$ are configured as $\langle 8,4,6\rangle$, and the remaining resources of server $C$ are configured as $\langle 7,2,9\rangle$; the maximum value of all kinds of resources is $\langle 10,6,9\rangle$, the next requirement $D$ is $\langle 6,3,4\rangle$, and then $P_{\mathrm{A}}=2.22, P_{\mathrm{B}}=2.13, P_{\mathrm{C}}=2.03$, and $P_{\mathrm{C}}<P_{\mathrm{B}}<P_{\mathrm{A}}$, but because the resource requirements are not met on $P_{\mathrm{C}}$, finally select server B to deploy D instance.

3.2. Resource Coordination Allocation Algorithm. Based on predicting the flow and the flow in a certain period $t$, and dynamically adjusting the NF instance according to the allocated and unused resources in the cluster server, for the newly arrived workflow, the unallocated resources are allocated according to the resource requirements. Cooperative and complementary resource allocation heuristics are aimed at reducing resource fragmentation and achieving high resource utilization. In the problem, the overall utilization is driven by the arrival rate and scheduling of the jobs. Our algorithm is inspired by the packing strategy in [25] and dominant resource idea in [11]. The algorithm first classifies the request flows life cycle and then uses the complementary advantages of the dominant resources to maximize the distance function for the resource requirements of the jobs, and the distance function is shown in the following equation:

$$
\sum_{r=1}^{|R|}\left(d_{i r}-d_{j r}\right)^{2}
$$

As shown in Figure 3, it represents four similar requests and server available resources and network function deployment. It can be seen that the resource requests for requests 1 and 2 are memory, and the resource requests for requests 3 and 4 are CPUs. It is calculated that the sum of the deviations of requests 1 and 3 is 10 , and the sum of the deviations of requests 1 and 4 is 13 , and then requests 1 and 4 are cooperatively packaged and requests 2 and 3 are cooperatively allocated. The allocation method adopts formulas (9) and (10). Detailed resource allocation algorithm is listed below for allocating unused resources to newly arrived jobs.

Input: network function service request set $S$, network function node set $F$, and various resource capabilities of different nodes $C_{l}=\left\{c_{l}^{1}, c_{l}^{2}, \ldots, c_{l}^{r}\right\}, \forall l \in[1,|L|]$.

Output: a viable resource allocation scheme that includes $x_{i j k}, \forall i \in[1,|S|], \forall j \in[1,|F|], \forall k \in V_{l}$.

Step 1: pretreatment: according to the request service set $S$, the service $s_{i}$ and its corresponding service function set $f_{i j}$ and the required resource quantity are obtained cyclically.

Step 2: requests are classified long-term and short-term according to the life cycle of the flow. For each type of request flow, the service request set $S$ is sorted into $S_{R}$ in descending order according to the resource type $r \in R$.

Step 3: the difference between the largest item in the resource type $S_{r}$ and the vector $S_{r}^{\prime}$ of other resource types is calculated, and the distance of each request is calculated according to equation (10); the items with the largest deviation are packaged and paired successively according to the calculation results.

Step 4: find the VM subset that meets the matching resource requirements in the function node set, calculate the corresponding value $P$ according to the formula (9), take the node with the smallest value, and select the nodes deploying the same type of stream as the distribution.

Step 5: if the job stream is long term which has a large percentage of jobs with small resource requirements, fit 


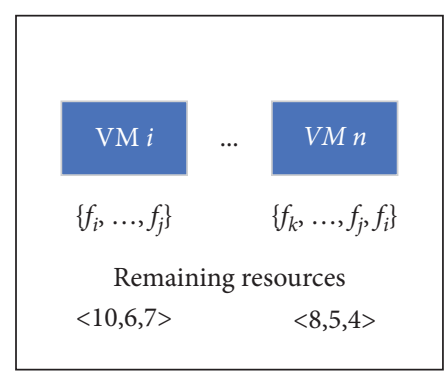

Request 1: $\left(<1,2,1>, f_{i}\right)$

Request 3: $\left(<4,1,1>, f_{j}\right)$

(a)

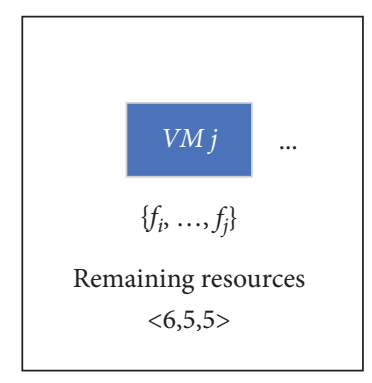

Request 2: $\left(<2,3,2>, f_{j}\right)$

Request 4: $\left(<4,2,3>, f_{i}\right)$

(b)

Figure 3: Network configuration and request instance. (a) Server 1. (b) Server 2.

into the system as many jobs as possible at a time; otherwise, fill the maximum jobs first according to equation (9).

Step 6: loop through the above steps until the assignment is complete.

\section{Experiments}

4.1. Preliminary. In this study, we use on-line traffic data on [26] to train and test the model. Each record is derived from .pcap files of the Skype traces that contain the features source address, destination address, time-stamp, request stream size, protocol, and other information from real users. The traces contain network traffic captured on main campus link that people have access [26], and the users can be administration staff, faculty members, or students. In each time slot, we regard the packets that have the same source and destination IP as a flow. The flow rate is calculated by dividing the total traffic size of the records in the flow by the interval of a time slot. We have extracted 290,148 network flows from the dataset. The data were split into training (70\%), validating (10\%), and testing (20\%). We have used Tensorflow to implement all the models and the python package scikit-learn to calculate performance metrics.

Research in [4] has proved that traffic load on NF instances brings remarkable overheads on virtual machine to the cloud proxy system. Moreover, a network flow consists of all packets sharing a unique bidirectional combination of source and destination IP addresses and transport protocol, etc. Based on the record of flows, the prediction of source and destination is used to infer the route of request flow and the corresponding service chain. Hence, here we examine the prediction accuracy of traffic flow and service chain. The forecast results will be the basis for providing the VNF instances and aggregating service chains to better utilize the limited and valuable resources over time.

Different from the previous service prediction model, we need to distinguish the type of flows. In each time slot, we regard the same source and destination pair as a type of NF service chain. In Figure 4, we replace the source and destination pairs of the request with numbers to represent the request flow and the figure shows a good prediction of 200

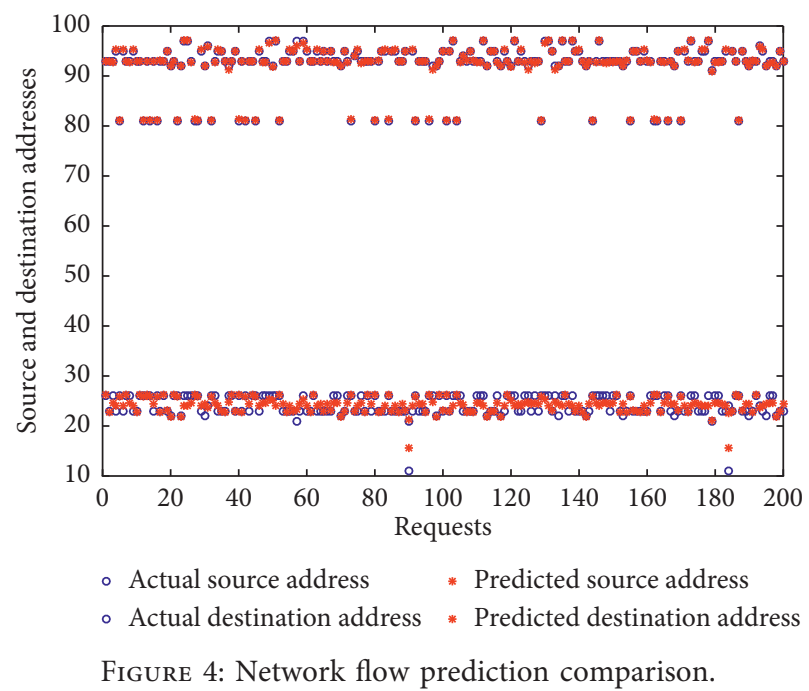

requests. Even if the result may have a certain range of deviation because the IP address is often divided according to the area, the deviation range is acceptable and controllable. Since the data set is captured on the campus main link, the addresses are regular. Hence, we will do more learning on network flow classification to detect much information about a current network flow in the future [27]; here, we focus on examining the prediction accuracy of traffic flow and the method can also be used for flow-type prediction. Before training, in order to have an intuitive understanding of the traffic fluctuations of flows over different time of the target data, we show the part of the traffic fluctuation values collected from 10 am in Figure 5 and part of the statistics and variation of number of requests per second in Figure 6. It shows that the provider must decide where (or whether) to place runnable tasks tens of times per second and even frequently needs to restart tasks. Hence, it is important to effectively estimate upcoming traffic rates and adjust VNF deployment.

Following the research in [28], in previous forecasting approaches, we know that multilayer perception (MLP) performed well in all individual models. In preliminary tests, several experiments are designed to compare with MLP to validate the effectiveness of the proposed LSTM RNN model in traffic flow prediction. After many experiments, we use 


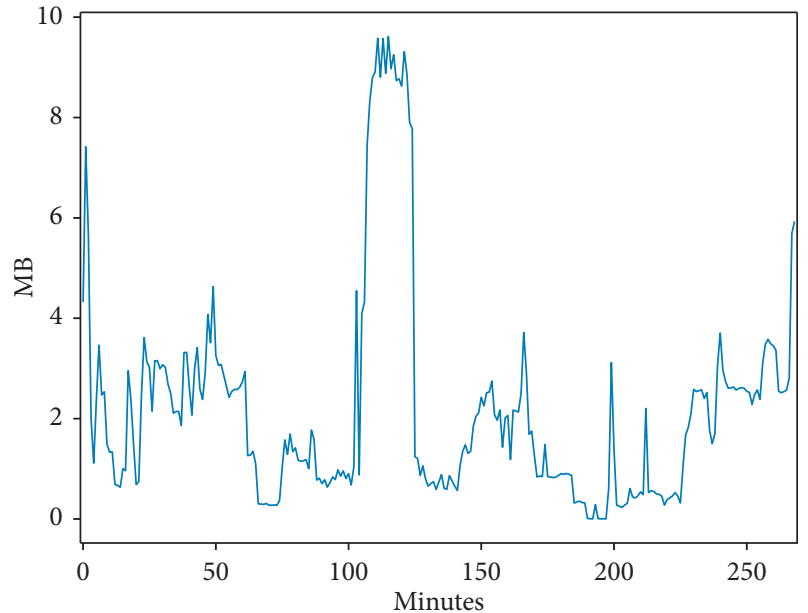

Figure 5: Traffic flow.

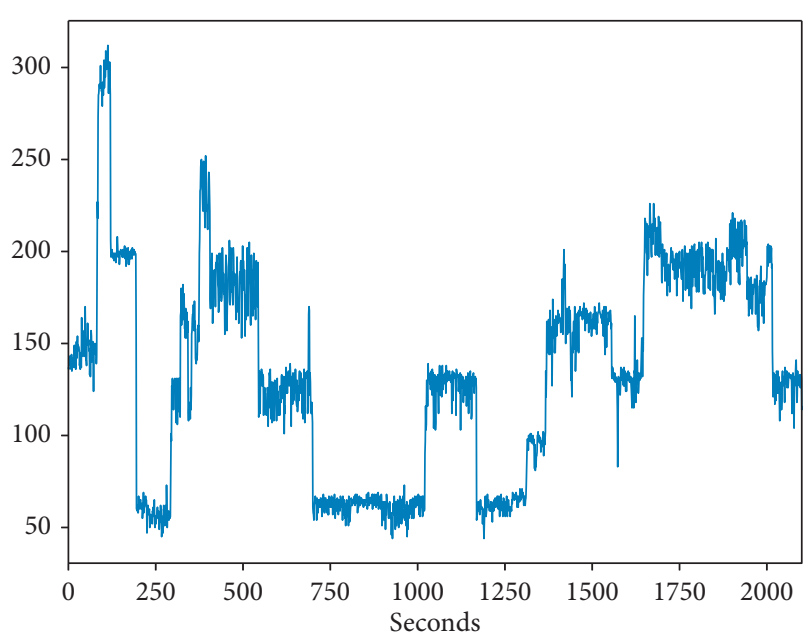

Figure 6: Statistics and variation of requests.

two hidden layers and the number of hidden units in each hidden layer is assigned to 64 and 32, respectively. In addition, the fully connected layers have been added to all models.

4.2. Validation and Analysis. The excellent performance of LSTM RNN mainly benefits from the memory ability. We illustrated the training phases' MLP and LSTM in Table 2 to verify the ability of LSTM RNN to memorize historical data. As we can see from Table 2, with the increase of input data length, the RMSE of LSTM model decreases faster than MLP at the start. When the input data length increases, the decline rates of both slows down, but compared with MLP, RMSE of LSTM RNN is lower and LSTM is relatively stable at a low level. Figure 7 shows the traffic flow comparison of observation values collected from 133.7 to 137.7 seconds, which contain more than 300 records, and prediction values obtained by LSTM RNN model. Intuitively, the prediction results are fairly good, and most of the fluctuations are captured. While previous forecasting approaches MLP [28], it shows a tendency to the fitting of
TABle 2: Prediction results of the RMSE.

\begin{tabular}{lcccc}
\hline Length & 1 & 10 & 20 & 30 \\
\hline MLP & 51.614 & 49.570 & 47.908 & 46.672 \\
LSTM & 47.186 & 39.860 & 339.552 & 39.776 \\
\hline
\end{tabular}

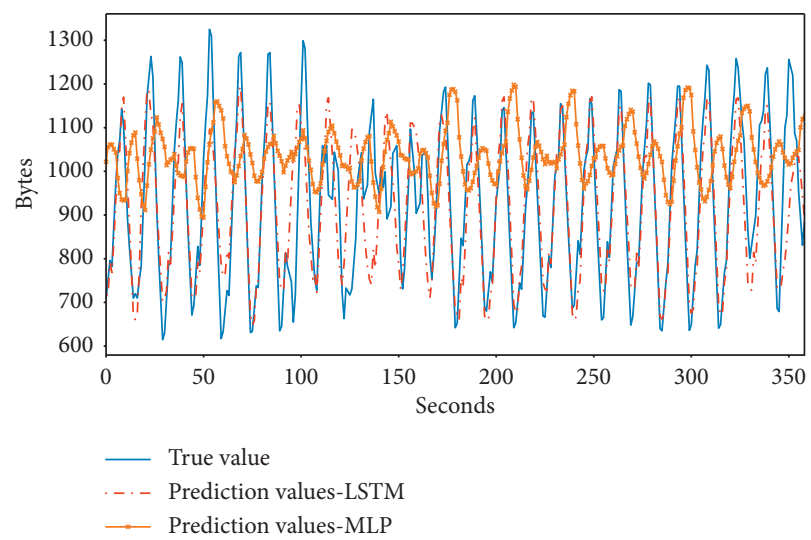

Figure 7: Comparison of observation and prediction traffic flow.

mean value. Figure 5 shows the flow fluctuates over time, which means that the traffic flow in the past prediction interval has a impact on the current traffic flow, but the impact of long historical data is relatively small, and LSTM RNN benefits from the memory ability. It is worth noting that a longer input history means more training rounds and time.

The generalization capability is an important evaluation criterion for a prediction model. However, the statistical result is about 10,000 flows per minute. We try to aggregate those records that have the same source and destination IP and fall into same time slot as a flow, but we found that it will destroy the time series of the data and seriously affect the accuracy of the training. Limited by computing power, in this study, we evaluated the generalization capability with different prediction intervals through the number of flows. The prediction intervals used are $10,20,30$, and 60 , and the sliding window lengths are 32 and 64 [29]. The prediction results are shown in Table 3. From Table 3, we can see that the RMSE rises with the increase of prediction horizon. But LSTM model is lower and less sensitive to prediction interval.

To evaluate the effectiveness of resource utilization, the comparison schemes are [7] and [30], and the former adopts a single resource allocation method, the latter aims at minimizing the standard deviation of all server loads. They minimize the system imbalance by different resources priority, and the priority is given based on their load distributions. Here, we have used MATLAB to simulate the configuration of the server CPU and memory resources in Table 1 and randomly set a set of virtual machine nodes in each server. In the case shown in Figure 8, we fixed the life time of the flow and compared the average utilization ratio of multiple resources of the virtual machines to show the improvement of resource utilization. In the experiments, we 
TABle 3: Prediction results of the RMSE.

\begin{tabular}{lcccc}
\hline Number & 10 & 20 & 30 & 60 \\
\hline MLP-32 & 56.71 & 60.09 & 60.86 & 76.61 \\
LSTM-32 & 50.93 & 52.67 & 52.75 & 54.85 \\
MLP-64 & 52.18 & 57.17 & 58.00 & 77.40 \\
LSTM-64 & 49.70 & 51.09 & 52.86 & 54.29 \\
\hline
\end{tabular}

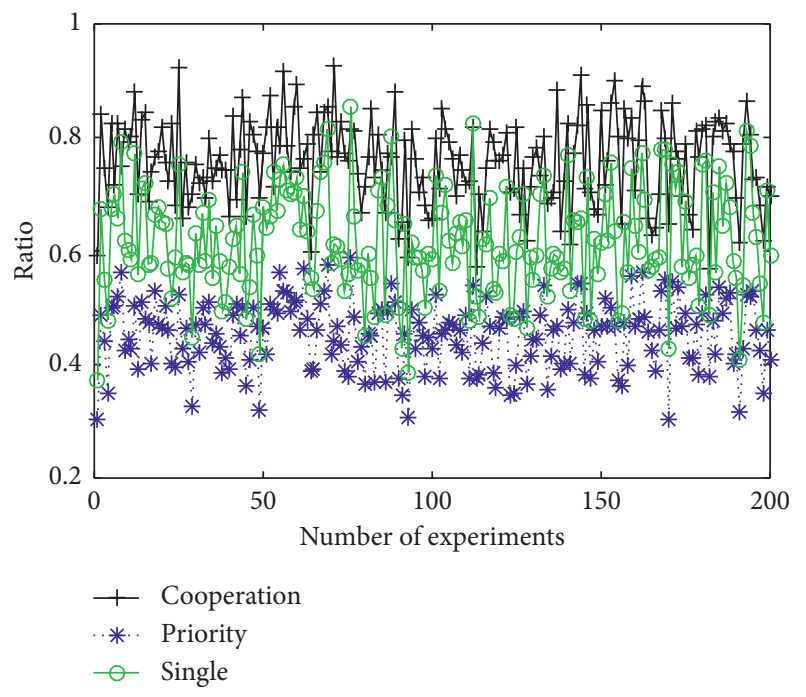

FIgURE 8: Resource allocation utilization.

set the demand quantity of different resource types for each request and gradually increased the number of service sets. From the results compared with [7] (denoted as Single) and [30] (denoted as Priority) we can find that the utilization improvement rate of collaborative resource allocation mode is mostly better. The resource utilization rate of cooperation showed better performance distribution than that of others in the heterogeneous setting resource allocation mode. The reason behind is that they are designed to achieve balanced load distribution, while ours is devised for reducing resource fragmentation according to the complementary strategies of different resource-type requirements. Even if Jia et al. [30] have adopted to migrate from the heaviest loaded node, the balance is based on the current load situation, without considering future resource requests, flow type, and dominant resources. In this study, LSTM is used to predict and use the resource and then dynamically allocate the resources to improve the resource utilization. The way of allocation of collaborative resources to jobs is consistent with our intuition; that is, the cooperative resource consideration mode is adopted in the allocation and the probability of virtual machine bottleneck resources in the server can be reduced under a certain probability so that the remaining space can be fully allocated to other newly arrived jobs and the resource utilization can be improved. Hence, cooperation results in a more balanced load distribution, and the performance variation of cooperation is adapted to traffic variations.

We verify the advantages of considering dynamic deployment and complementary resource allocation above, and here, we regard VM as a slot. In Figure 9, ratio 1

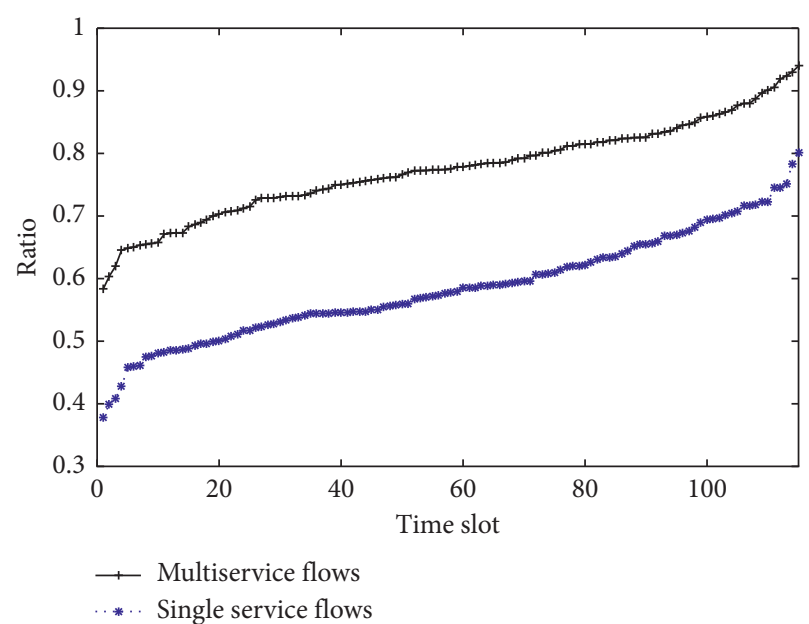

Figure 9: Comparison of different service flow scenarios

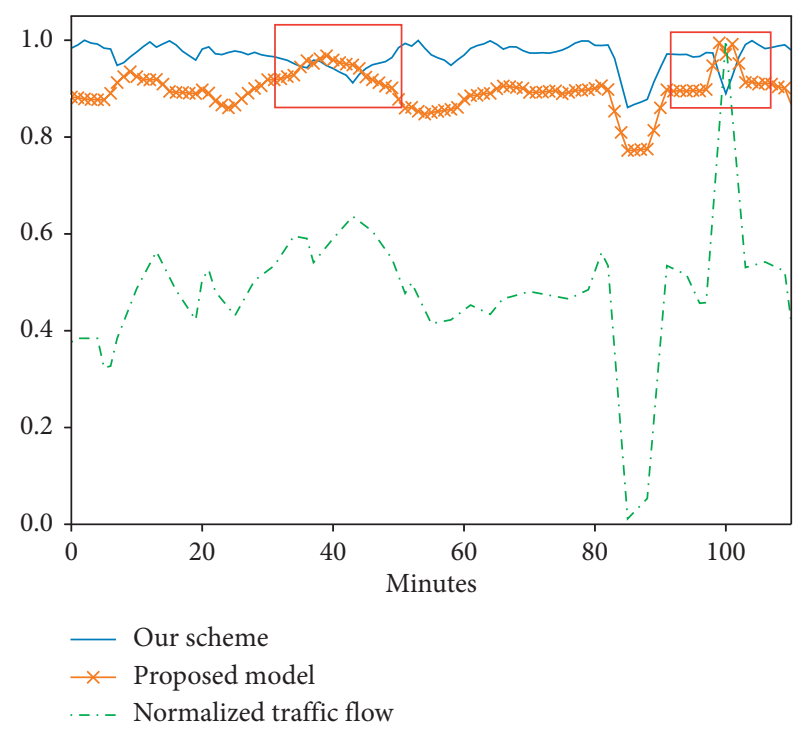

FIgURE 10: Utilization of VMs allocated.

corresponds to a perfect match between the task assignment and the slot, and ratio less than 1 corresponds to the ratio of underutilized resources. For more than 100 time periods, we use the method in [31] to set a $10 \%$ long-term business flow. As shown in the figure, the multiservice flow approach provides higher resource utilization. This is because it better matches the resource allocation with the task demand cycle. Short-cycle flow typically consists of a single execution phase; on the contrary, the completion time of long-term workflows consists of many phases. Therefore, among the multiple service flows, the small work completion time is fast and improves the response time of the small jobs without impacting the large jobs, and the higher resource utilization can be achieved. Figure 10 depicts the utilization when VMs are allocated based on our scheme and the method proposed in [32], in which dynamic scaling of resources in NFV is done by combining the offline method by varying the window size along with different types of days and the online method ESA (Exponential Smoothing Average) to detect the 
load transition tendency. From the figure, we observe [32] performs well on dealing with peaks, which benefit from the ESA detects the peaks quickly, but in the long run, our approach performs stable and better. This is partly because our approach considers multiple resource coordination and flow types, which reduce the resource fragmentation and the lifetime limitation of the flows, thereby improving valuable network resource utilization.

\section{Conclusion}

This paper proposes a deep learning-based forecasting strategy based on the dynamic adjustment of network function instances in network function virtualization resource allocation in cloud services and then designs a collaborative strategy based on deep dynamic learning. The scheme realizes reasonable expansion and allocation of virtual network resources, effectively reducing resource fragmentation and flow life cycle constraints, thereby improving valuable network resource utilization. Finally, the experimental results based on actual data show that this research can effectively improve the ability of virtualized network function resources adjustment and effectively improve the utilization of network resources.

\section{Data Availability}

The datasets have been shared on the public website http:// tstat.polito.it/traces-skype.shtml.

\section{Conflicts of Interest}

The author declares that there are no conflicts of interest.

\section{Acknowledgments}

The author would like to thank Wendong Wang, Xiangyang Gong, and Xirong Que, State Key Laboratory of Networking and Switching Technology, BUPT, for their valuable support.

\section{References}

[1] J. G. Herrera and J. F. Botero, "Resource allocation in NFV: a comprehensive survey," IEEE Transactions on Network and Service Management, vol. 13, no. 3, pp. 518-532, 2016.

[2] R. Guerzoni, "Network functions virtualisation: an introduction, benefits, enablers, challenges and call for action, introductory white paper," in Proceedings of the SDN and OpenFlow World Congress, pp. 1-16, Darmstadt, Germany, October 2012.

[3] M. Leconte, G. S. Paschos, P. Mertikopoulos, and U. C. Kozat, "A resource allocation framework for network slicing," in Proceedings of the IEEE INFOCOM 2018-IEEE Conference on Computer Communications, pp. 2177-2185, Honolulu, HI, USA, April 2018.

[4] H. Wang, T. Li, R. Shea et al., "Toward cloud-based distributed interactive applications: measurement, modeling, and analysis," IEEE/ACM Transactions on Networking, vol. 26, no. 1, pp. 3-16, 2018.

[5] NEC/Netcracker NaaS, https://www.netcracker.com/naas.
[6] C. Reiss, A. Tumanov, G. R. Ganger et al., "Heterogeneity and dynamicity of clouds at scale: Google trace analysis," in Proceedings of the Third ACM Symposium on Cloud Computing, San Jose, CA, USA, 2012.

[7] X. Fei, F. Liu, H. Xu et al., "Adaptive VNF scaling and flow routing with proactive demand prediction," in Proceedings of the IEEE INFOCOM 2018-IEEE Conference on Computer Communications, pp. 486-494, Honolulu, HI, USA, April 2018.

[8] V. Jalaparti, I. Bliznets, S. Kandula et al., "Dynamic pricing and traffic engineering for timely inter-datacenter transfers," in Proceedings of the 2016 ACM SIGCOMM Conference, pp. 73-86, Florianopolis, Brazil, August 2016.

[9] Z. Xiao, W. Song, and Q. Chen, "Dynamic resource allocation using virtual machines for cloud computing environment," IEEE Transactions on Parallel and Distributed Systems, vol. 24, no. 6, pp. 1107-1117, 2013.

[10] A. Bremler-Barr, Y. Harchol, and D. Hay, "OpenBox: a software-defined framework for developing, deploying, and managing network functions," in Proceedings of the 2016 conference on ACM SIGCOMM 2016 Conference, pp. 511-524, Florianopolis, Brazil, August 2016.

[11] A. Ghodsi, M. Zaharia, B. Hindman et al., "Dominant resource fairness: fair allocation of multiple resource types," Nsdi, vol. 11, p. 24, 2011.

[12] J. Liu and H. Shen, "Dependency-aware and resource-efficient scheduling for heterogeneous jobs in clouds," in Proceedings of the Cloud Computing Technology and Science (CloudCom), 2016 IEEE International Conference on, pp. 110-117, Luxembourg City, Luxembourg, December 2016.

[13] Y. Chen, S. Alspaugh, and R. Katz, "Interactive analytical processing in big data systems: a cross-industry study of mapreduce workloads," Proceedings of the VLDB Endowment, vol. 5, no. 12, pp. 1802-1813, 2012.

[14] L. Qu, C. Assi, and K. Shaban, "Delay-Aware scheduling and resource optimization with network function virtualization," IEEE Transactions on Communications, vol. 64, no. 9, pp. 3746-3758, 2016.

[15] X. Zhang, C. Wu, Z. Li, and F. C. M. Lau, "Proactive vnf provisioning with multi-timescale cloud resources: fusing online learning and online optimization," in Proceedings of the IEEE INFOCOM 2017-IEEE Conference on Computer Communications, pp. 1-9, Atlanta, GA, USA, May 2017.

[16] X. Wang, C. Wu, F. Le, A. Liu, Z. Li, and F. Lau, "Online VNF scaling in datacenters," in Proceedings of the 2016 IEEE 9th International Conference on Cloud Computing (CLOUD), pp. 140-147, San Francisco, CA, USA, June 2016.

[17] V. Eramo, E. Miucci, M. Ammar, and F. G. Lavacca, "An approach for service function chain routing and virtual function network instance migration in network function virtualization architectures," IEEE/ACM Transactions on Networking, vol. 25, no. 4, pp. 2008-2025, 2017.

[18] F. Xiao, Z. Lin, Y. Sun, and Y. Ma, "Malware detection based on deep learning of behavior graphs," Mathematical Problems in Engineering, vol. 2019, Article ID 8195395, 10 pages, 2019.

[19] Y. Tian and L. Pan, "Predicting short-term traffic flow by long short-term memory recurrent neural network," in Proceedings of the 2015 IEEE International Conference on Smart City/ SocialCom/SustainCom (SmartCity), pp. 153-158, Chengdu, China, December 2015.

[20] T. T. T. Nguyen and G. J. Armitage, "A survey of techniques for internet traffic classification using machine learning," 
IEEE Communications Surveys and Tutorials, vol. 10, no. 1-4, pp. 56-76, 2008.

[21] B. Ng, M. Hayes, and W. K. G. Seah, "Developing a traffic classification platform for enterprise networks with SDN: experiences \& lessons learned," in Proceedings of the IFIP Networking Conference (IFIP Networking), pp. 1-9, Toulouse, France, May 2015.

[22] C. Chekuri and S. Khanna, "On multidimensional packing problems," SIAM Journal on Computing, vol. 33, no. 4, pp. 837-851, 2004.

[23] G. Zhang, B. Eddy Patuwo, and M. Y. Hu, "Forecasting with artificial neural networks:" International Journal of Forecasting, vol. 14, no. 1, pp. 35-62, 1998.

[24] S. Hochreiter and J. Schmidhuber, "Long short-term memory," Neural Computation, vol. 9, no. 8, pp. 1735-1780, 1997.

[25] W. Leinberger, G. Karypis, and V. Kumar, "Job scheduling in the presence of multiple resource requirements," in Proceedings of the 1999 ACM/IEEE conference on Supercomputing (CDROM), p. 47, Portland, USA, November 1999.

[26] TCP statistic and analysis Tool: Skype traces, http://tstat. polito.it/traces-skype.shtml.

[27] M. Lopez-Martin, B. Carro, A. Sanchez-Esguevillas, and J. Lloret, "Network traffic classifier with convolutional and recurrent neural networks for internet of things," IEEE Access, vol. 5, pp. 18042-18050, 2017.

[28] C. Katris and S. Daskalaki, "Comparing forecasting approaches for Internet traffic," Expert Systems with Applications, vol. 42, no. 21, pp. 8172-8183, 2015.

[29] A. Sang and S.-Q. Li, "A predictability analysis of network traffic," Computer Networks, vol. 39, no. 4, pp. 329-345, 2002.

[30] Y. Jia, I. Brondino, R. J. Peris et al., "A multi-resource load balancing algorithm for cloud cache systems," in Proceedings of the 28th Annual ACM Symposium on Applied Computing, pp. 463-470, Salamanca, Spain, 2013.

[31] P. Delgado, F. Dinu, A. M. Kermarrec et al., "Hawk: hybrid datacenter scheduling," in Proceedings of the 2015 USENIX Annual Technical Conference, USENIX Association, Berkeley, CA, USA, July 2015.

[32] A. Bilal, T. Tarik, A. Vajda, and B. Miloud, "Dynamic cloud resource scheduling in virtualized $5 \mathrm{~g}$ mobile systems," in Proceedings of the 2016 IEEE Global Communications Conference (GLOBECOM), pp. 1-6, Washington, DC, USA, December 2016. 